\title{
Effect of Thermo Diffusion on Mass And Heat Transfer Flow on Convective Viscous Electrically Conducting Fluid Through a Porous Medium Bounded by a Semi-Infinite Vertical Plate with Variable Electrically Conductivity Diffusion Thermo Chemical Reaction
}

\section{T. Siva Nageswara Rao}

\begin{abstract}
In this paper we investigate thermochemical diffusion reaction and effects of thermal diffusion on the mass and heat transfer flux Although the effects of thermal diffusion are vast, they can be transmitted through a very wide medium.. By employing Galerkin-finite element analysis the equations solved with three nodded line segments
\end{abstract}

Keywords:, Chemical reaction, Electrical conductivity, Thermo-Diffusion, Mass and Heat Transfer

\section{INTRODUCTION}

The combination of concentration and temperature gradients in the liquid leads to floating currents. In the presence of the radiative currents of a high temperature electrically conductive fluid, the magnetic field play a key role in many engineering, industrial and environmental processes. fossil fuel combustion processes, Heating and cooling rooms, Evaporation from large open water reservoirs, astrophysical flows, solar power technology and space vehicle re-entry. More apps and better understanding of this topic is given by Rashad [1], Sanyal and Adhikari [2], Muthucumaraswamy and Kulandaivel [3], Prasad and Reddy [4], Singh and Kumar [5] and Raptis and Perdikis [6]. Chamkha [7] considered over an accelerating semiinfinite plate with hydromagnetic boundary layer flow

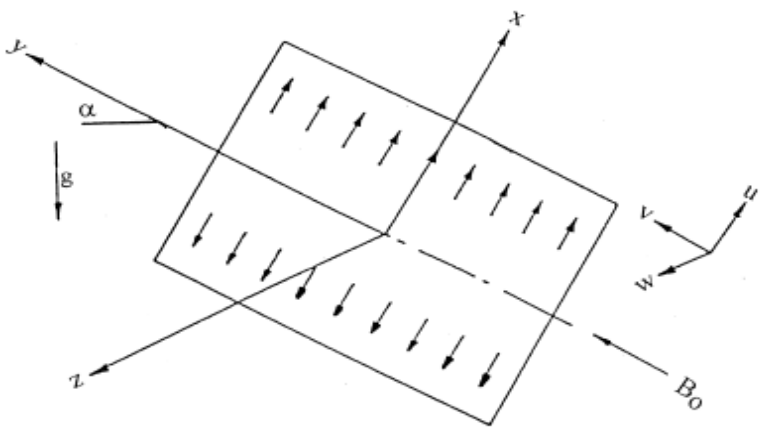

Fig. 1. The flow model in schematic diagram

Revised Manuscript Received on December 15, 2019

T. Siva Nageswara Rao, Division of Mathematics, Vignan's Foundation for Science Technology \& Research (Deemed to be University), Vadlamudi, Guntur (Dt.), A.P, India

\section{PROBLEM FORMULATION}

In this Constant, laminar composite mass and heat transfer through a natural convection along a continuously moving semi-infinite permeable flat plate, inclined at a sharp angle from the vertical along the plate $\mathrm{x}$-axis measured, For the normal to the direction of flow, in the direction of $\mathrm{y}, \mathrm{Bo}$ is magnetic field of consistent strength applied, this is normal to the flow direction . on the plate surface Fluid suction is imposed. For allowing possible heat generation effects within the flow heat source is placed. Newtonian fluid is considered, heat generating and electrically conducting. At $\mathrm{T}_{\mathrm{w}}$ the surface temperature of is to be uniform which is more than $T_{\infty}$. At $C_{w}$ the surface is maintained species concentration uniform and $\mathrm{C}_{\infty}$ the ambient fluid which are vanishes to help For understanding the atmosphere through the delivery of mass to the surface by non-precipitation the out-turn of thermophotesis are cosidered.

The equations governing the heat and mass transfer are:

$$
\frac{\partial u}{\partial x}=-\frac{\partial v}{\partial y}
$$

(Continuity Equation)

$u_{x} u+v_{y} v=\frac{\partial^{2} u}{\partial y^{2}} v+\beta\left(T-T_{\infty}\right) g \cos \alpha-\beta^{\bullet}\left(C-C_{\infty}\right) g \cos \alpha-u \frac{\sigma B_{0}^{2}}{\rho}-u\left(\frac{\mu}{k}\right)$

(Equation of Momentum )

$\frac{\partial T}{\partial y} v+\frac{\partial T}{\partial x} u=\frac{\partial^{2} T}{\partial y^{2}} \frac{\lambda_{g}}{\rho c_{p}}+K_{12} \frac{\partial^{2} C}{\partial y^{2}}-\frac{\partial\left(q_{R}\right)}{\partial y}$

(Energy Equation )

$C_{x} u=-v C_{y}+C_{y y} D-\left(V_{T} C\right) \partial_{y}+k_{11} T_{y y}-k_{1}^{\prime} C$

(Diffusion Equation )

Here $u, v$ are rate of change in displacement elements in the direction of $x$ and $y$, the kinematic viscosity is $v$, Thermophoretic velocity $V_{T}$, the a substance that continually deforms $\lambda_{g}$, the acceleration of gravity $g$, at constant pressure the specific heat $c_{p}$, the fluid temperature in the free stream, the temperature of plate, the temperature of the fluid inside the thermal limitation are $T_{\infty}, T$ and $T_{w}$, the magnetic induction $B_{0}$,

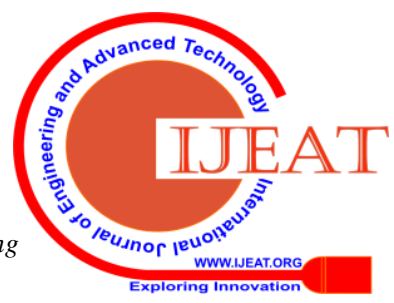




\section{Effect of Thermo Diffusion on Mass And Heat Transfer Flow on Convective Viscous Electrically Conducting Fluid Through a Porous Medium Bounded by a Semi-Infinite Vertical Plate with Variable Electrically Conductivity Diffusion Thermo Chemical Reaction}

the gradient in the concentration of the species and the molar flux due to molecular diffusion is $D$, , the cross diffusivities are $\mathrm{k}_{11}, \mathrm{k}_{12}$, the density of the fluid $\rho$, the concentrations are $C, C_{w}$ and $C_{\infty,}$, the the degree to which a specified material conducts electricity $\sigma$, and the volumetric constant $\beta$.

The boundary conditions are:

$$
v= \pm v_{w}, u=U_{0}, C=C_{w}=0, T=T_{w} \quad \text { at } \quad y=0
$$

$C=C_{\infty}, \quad T=T_{\infty}, u=0 \quad$ at $\quad y \rightarrow \infty$

here the connected pore spaces are to one another $v_{w}(x)$ represents its pointer indicates blowing $(>0)$ or suction $(<0)$ and the invariable plate velocity $U_{0}$. Here our notice to consider that $v_{w}(x)$, for these the fluid is suctioned through the porous surface the transpiration function variable of the order $x^{-1 / 2}$.

After using Rosseland diffusion approximation, the heat flux due to radiative is given by

$q_{r}=-\frac{\partial T^{\prime 4}}{\partial y} \frac{4 \sigma^{\bullet}}{3 \beta_{R}}$

(6a)

and by Taylor's expansion we obtain

$T^{\prime 4} \cong 4 T_{e}^{3} T-3 T_{e}^{4}$ after not considering higher order terms.

$\sigma^{\bullet}$ is the Stefan-Boltzman constant and $\beta_{R}$ is the mean absorption coefficient.

we initiate the following dimensionless variables for obtain likeness solution of the problem:

$$
\eta=y \sqrt{\frac{U_{0}}{2 v x}}, \quad \psi=\sqrt{2 v x U_{0}}, f(\eta) \theta(\eta)=\frac{T-T_{\infty}}{T_{w}-T_{\infty}}, \phi(\eta)=\frac{C}{C_{\infty}}
$$

(7a)



(a)
The divergence-free function $\psi$, it satisfies the equation (1). Beacause $u=\frac{\partial \psi}{\partial y}$ and $v=\psi_{x}$, we have

$v=\sqrt{\frac{v U_{0}}{2 x}}\left(f-\eta f^{\prime}\right)$ and $u=U_{0} f^{\prime}$

(7b)

The powers denotes ordinary differentiation with respect to $\eta$.

$f^{\prime \prime \prime}+f f^{\prime \prime}+G r(\theta+\mathrm{N} \phi) \cos \alpha-\alpha_{l} \theta-\left(M^{2}+D^{-1}\right) f^{\prime}=0$

$(8)$

$\theta^{\prime \prime}\left(1+4 / 3 \mathrm{~N}_{1}\right)+\operatorname{Pr} \mathrm{f} \theta^{\prime}=0$

(9)

$\phi^{\prime \prime}+\operatorname{Sc}\left(f-\tau \theta^{\prime}\right) \phi^{\prime}-\operatorname{Sc} \tau \phi \theta^{\prime \prime}=-\operatorname{ScSo} \theta^{\prime \prime}-\gamma \theta$

(10)

The conditions specified for the $(5 \mathrm{a}, 5 \mathrm{~b})$ then convert into

$f^{\prime}=1, \quad \phi=0, \quad \theta=1, \quad f=f_{w}, \quad$ at $\quad \eta=0$

(11a)

$f^{\prime}=1, \quad \theta=0, \quad \phi=1 \quad$ at $\quad \eta \rightarrow \infty$

(11b)

where the accusatorial wall mass spread coefficient $f_{w}=-v_{w}(x) \sqrt{\frac{2 x}{v U_{0}}}$ such that $f_{w}>0$ stipulate wall suction and wall injection for $f_{x}<0$.

\section{EMPIRICAL STUDY}

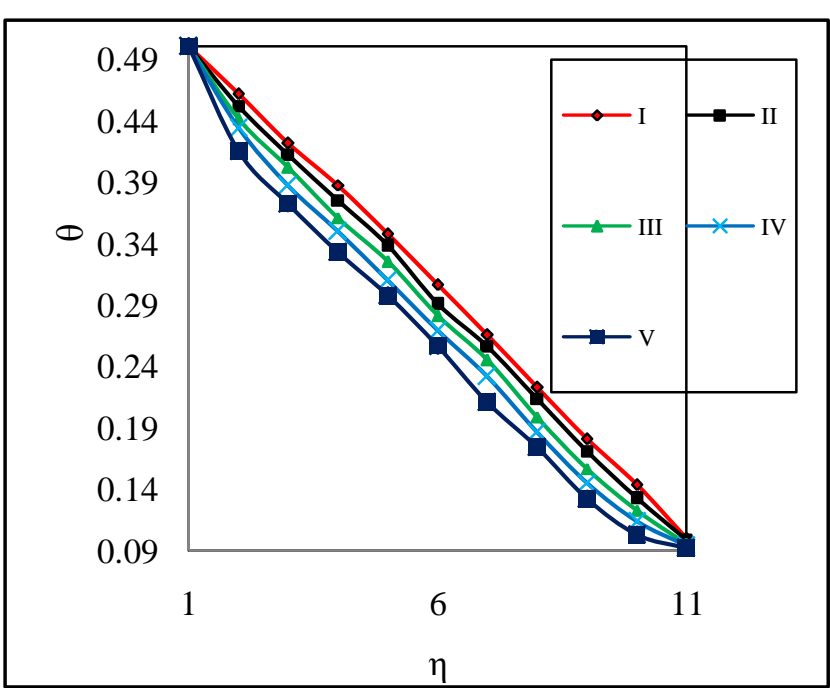

(b) 


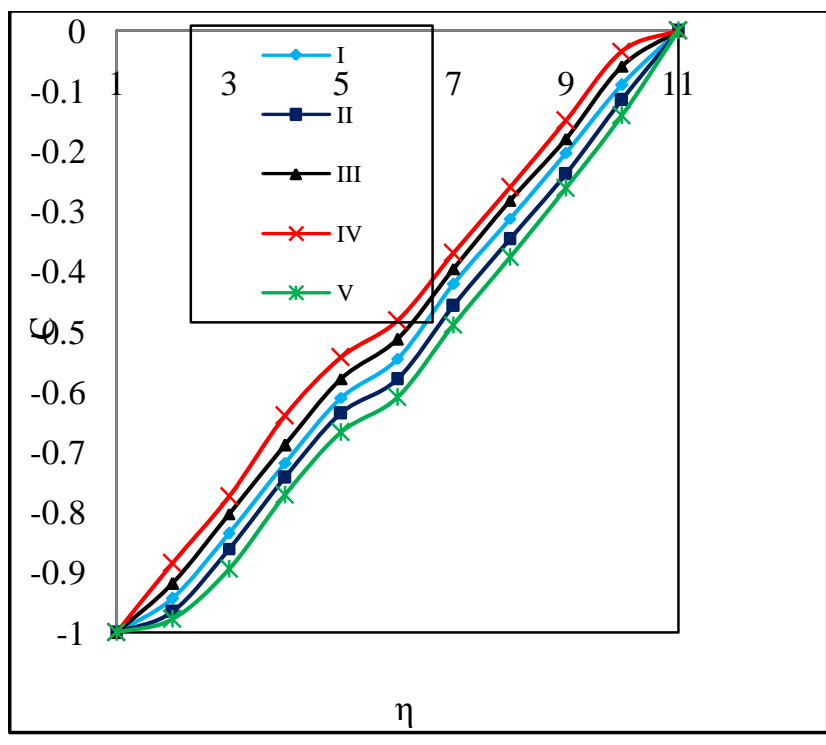

(c)

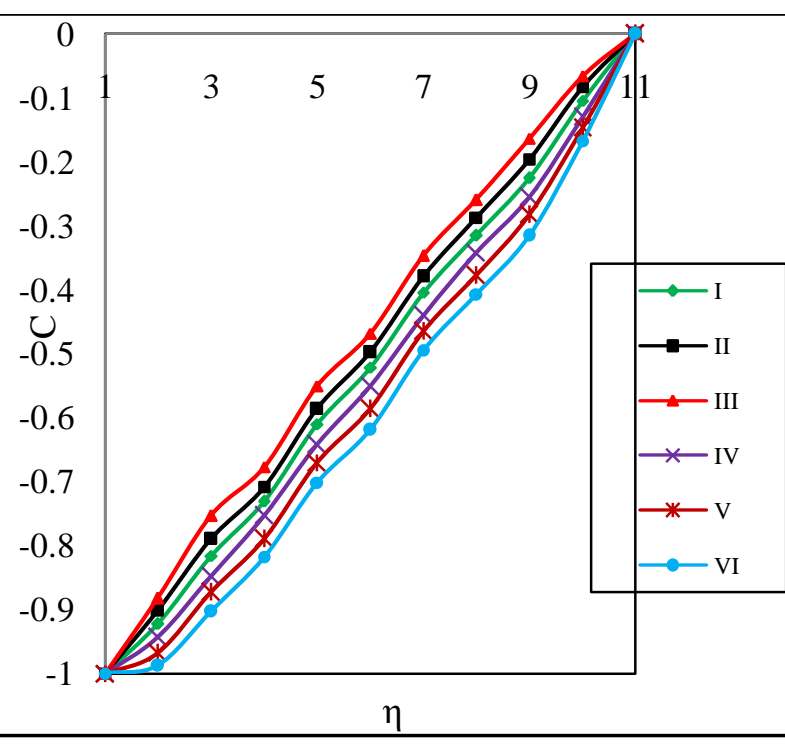

(d)

Fig.2. The change of $\mathrm{C}$ with $\alpha$ and $\lambda$, disparity of $\theta$ accompanied by $\alpha$ and $\lambda$

\begin{tabular}{|c|c|c|c|c|c|c|c|c|c|c|c|c|}
\hline & I & II & III & IV & $\mathrm{V}$ & & $\mathrm{i}$ & ii & iii & iv & $\mathrm{v}$ & vi \\
\hline$\alpha$ & $\frac{\pi}{6}$ & $\frac{\pi}{4}$ & $\frac{\pi}{3}$ & $\frac{\pi}{2}$ & $-\pi$ & $\lambda$ & $\frac{1}{2}$ & $\frac{3}{2}$ & $\frac{5}{2}$ & $-\frac{1}{2}$ & $-\frac{3}{2}$ & $-\frac{5}{2}$ \\
\hline
\end{tabular}

We notice that the original temperature $\theta$ reduces with increasing in $\lambda \leq 1.5$ and increase with higher than $\lambda \geq 2.5$ while it enhances with $|\lambda| \leq 1.5$ and depreciates with $|\lambda| \geq 2.5$ fig.(a). From $\operatorname{Fig}(\mathrm{b})$, the variation of $\theta$ with inclination $\alpha$ of the flat plate. It is noticed that an elevation in $\alpha \leq \frac{\pi}{4}$ depreciates in $\theta$ and for higher $\alpha \geq \frac{\pi}{3}$ we observed an improvement in the $\theta$. we determine the enhancement in the $C$ with rise of $\lambda>0$, reduces with $|\lambda|$ fig.(c). From fig.d the variation of $\mathrm{C}$ with inclination $\alpha$ of the plate shows that the original concentration depreciates with increase $|\alpha| \leq 4$ and all values of $\alpha$ lying in the interval $\left[\frac{\pi}{3}, \frac{\pi}{2}\right]$ we notice increase in the $\mathrm{C}$ and for still higher inclination $\alpha=\pi$ we find a depreciation in the $C$

\section{COMPARISON}

In this present study,we compared the results obtained here with the results of Prasad VR, Reddy NB[2008b] without $\mathrm{Nu}(\mathrm{N}=0)$ and $\gamma$ and compared with the outcomes of Chamkha [7] without $\mathrm{Q} 1=0$, the consequence are consensus.
Similarities between Shear stress, mass, heat transfer

Existing Results $(D u=$ Chamkha [2000] Results $\mathbf{0 ,} \mathbf{N}=\mathbf{0})$

\begin{tabular}{ccccccccc}
\multicolumn{10}{c}{$\mathbf{0 , \mathbf { N } = \mathbf { 0 } )}$} \\
$\gamma$ & 1 & 2 & -0.5 & - & 1 & 2 & -0.5 & -0.8 \\
& & & & 0.8 & & & & \\
$\alpha$ & 0.5 & 0.5 & 0.5 & 0.5 & 0.5 & 0.5 & 0.5 & 0.5 \\
$\mathrm{~N}$ & 61.1 & 52.5 & 10.1 & 9.8 & 61.1 & 52.5 & 10.2 & 9.86 \\
$\mathrm{u}$ & 23 & 63 & 14 & 60 & 251 & 23 & 14 & 7 \\
$\mathrm{~S}$ & 21.9 & 20.6 & 3.35 & 4.1 & 21.9 & 20.4 & 3.37 & 4.13 \\
$\mathrm{~h}$ & 44 & 30 & 62 & 56 & 44 & 30 & 92 & 5 \\
$\tau$ & 26.5 & 21.1 & 4.69 & 5.1 & 26.5 & 21.1 & 4.69 & 51.7 \\
& 05 & 30 & 30 & 82 & 16 & 16 & 30 & 92
\end{tabular}

\section{CONCLUSIONS}

The transverse velocity ( $f$ ), an increase in $\lambda>0$ we found that $|f|$ increased and with $\lambda<0$, depreciates. With respect to the $D^{-1}$ ( Darcy parameter) the change in $f$, that lower the penetrable parameter of tiny holes larger $|f|$ in the region fluid stream. The change of $f$ with respect to $\gamma$ we find that in the degenerating reaction case $|f|$ reduces and in generating decomposition case $\left|f^{\prime}\right|$ decreases with increase in $|\gamma| \leq 1.5$ and increases with $|\gamma| \geq 2.5$. An increase in the inclination $\alpha \leq \frac{\pi}{3}$ of 
the wall depreciates $\left|f^{\prime}\right|$ and enhances with higher $\alpha \geq \frac{\pi}{2}$

\section{REFERENCES}

1. Rashad AM. Perturbation analysis of radiative effect on free convection flows in porous medium in the presence of pressure work and viscous dissipation. Communication in nonlinear science and Numerical Simulation. 2009; 14: 140-153.

2. Sanyal DC, Adhikari A. Effect of radiation on MHD vertical channel flow. Bulletin of Calcutta Mathematical Society. 2006; 98(5): 487497.

3. Muthucumaraswamy R, Kulandaivel T. Radiation effects on moving vertical plate with variable temperature and uniform mass diffusion. Journal of Energy, Heat and Mass Transfer. 2008; 30: 79-88.

4. Prasad VR, Reddy NB. Radiation effects on an unsteady MHD convective heat and mass transfer flow past a semi-infinite vertical plate embedded in porous media. Journal of Energy. Heat and Mass Transfer. 2008b; 30: 57-78.

5. Singh KD, Kumar Rakesh. An Exact solution of an oscillatory MHD flow through a porous medium bounded by rotating porous channel in the presence of Hall current. International Journal of Applied Math \& Mechanics.2010; 6:28-40.

6. Raptis A, Perdikis C. Radiation and free convection flow past a moving plate. International Journal of Applied Mechanics and Engineering. 1999; 4: 817-821.

7. Chamkha AJ. Thermal radiation and buoyancy effects on hydrodynamic flow over an accelerated permeable surface with heat source or sink. International Journal of Engineering Science. 2000; 38: $1699-1712$ 